\author{
MAGNETIC FIELD CALCULATION FOR \\ FERMILAB-STYLE MAGNET COIL END
}

Kenji Ishibashi and A.D.McInturff

September 1982

A simple end field calculation is described, which utilizes a reciprocal theorem of mutual inductance between actual windings and a virtual coil. The calculation method is applied to a Fermilab-style magnet, and the computation results are compared with those obtained by GFUN.

\title{
INTRODUCTION
}

Quench of superconducting magnets usually takes place at the high field position in the magnet ends after training has ended. The magnet end windings are therefore located out of the iron yoke to remove the field enhancement of the iron. It is necessary in magnet design, therefore, to calculate the magnetic field in the end winding. The computer code GFUN developed by the Rutherford Laboratory Group, headed by W.Trowbridge, has the capability of three dimensional (3D) field calcultion. The code is a convenient program for general magnet design, and particularly 3D computation. Unfortunately, however, GFUN was not made to be completely applicable to Fermilab-style keystone conductor wound around circular apertures and the resulting end winding configurations, and consequently requires an approximation of winding configuration to compute it. This approximation may introduce some error in field computed.

There is another approach for the center axis field calculation which is a flux theorem ${ }^{1}$ for calculation of magnet ends. The theorem is based on mutual inductance between actual coils and infinitely long virtual cos $\theta$ winding, and converts $3 \mathrm{D}$ field information into two dimensional (2D) field integral along the magnet axis. Unlike 
computation based on Biot-Savart's law, ${ }^{2}$ this way does not need any tedious consideration of relative orientation of current vector to a location concerned. In the present report, this mutual inductance method was attempted to extend to calculation of the $3 \mathrm{D}$ field for the purpose of easily taking into account the exact configuration of the magnet ends, and the results computed were compared with those from the above geometry configuration by GFUN.

\section{RECIPROCAL THEOREM}

A simple example is derived to explain the principle of field calculation based on mutual inductance. Suppose that there is a coil $\alpha$ on $X-Y$ plane in Figure $1(a)$, and a field $B_{z}$ parallel to $z$ direction at location $\left(\mathrm{X}_{0}, \mathrm{Y}_{0}, \mathrm{Z}_{0}\right)$ is calculated. If a virtual coil $\beta$ with area a and current $I_{0}$ is assumed to be present, as shown in Figure $l(b)$, it would induce a flux $\Phi_{0}$ which penetrates the coil $\alpha$. Then, the mutual inductance $M$ between coils $\alpha$ and $\beta$ can be written as $M=\Phi_{0} / I_{0}$.

On the other hand, the current I on the coil will induce a flux $\Phi_{1}$ on the coil $\beta$. Because of a reciprocal theorem, the flux $\Phi_{1}$ should be given as $\Phi_{1}=$ MI by means of the above mutual inductance M. Therefore, the average field $B_{z}$ on the $\operatorname{coil} \beta$ is written as

$$
\begin{aligned}
B_{z} & =\Phi_{1} / a \\
& =I \cdot \Phi_{0} / I_{\theta} a
\end{aligned}
$$

When the area "a" of the coil " $\beta$ " becomes infinitesimal, the coil " $\beta$ " is considered to give the same field on the coil $\alpha$ as that of a magnetic dipole moment $\mu_{0} a I_{0}$, and the flux $\Phi_{0}$ in the coil " $\alpha$ " may be expressed as $\Phi_{0}=\mu_{0} a I_{0} \cdot \Phi_{z}$, where $\Phi_{z}$ stands for a flux which is induced by unit dipole moment and generated in the coil " $\alpha$ ". Eq. (1) is therefore given as 


$$
B_{z}=\mu_{0} I \Phi_{z}
$$

The unit magnetic dipole moment at the location $\left(\mathrm{X}_{0}, \mathrm{Y}_{0}, \mathrm{Z}_{0}\right)$ gives the following scaler potential, $\psi_{z}$ and then field $B_{z_{0}}$ perpendicular to the $X-Y$ plane:

$$
\begin{aligned}
& \psi_{z}=-\frac{1}{4 \pi} \frac{\partial}{\partial z} \frac{1}{\ell} \\
& B_{z_{0}}=\frac{\partial \psi_{z}}{\partial z}=\frac{1}{4 \pi} \frac{\partial}{\partial z}\left(\frac{\partial}{\partial z} \frac{1}{\ell}\right)
\end{aligned}
$$

where $\quad l=\left(\left(\mathrm{X}-\mathrm{X}_{0}\right)^{2}+\left(\mathrm{Y}-\mathrm{Y}_{0}\right)^{2}+\left(\mathrm{Z}-\mathrm{Z}_{0}\right)^{2}\right)^{\frac{1}{2}}$

Since $\Phi_{z}$ is given by integration of Eq. (4) over the area $A$ of the coil $\alpha$, the Eq. (2) is expressed as

$$
B_{z}=\frac{\mu_{0} I}{4 \pi} \int_{A} \frac{\partial}{\partial z}\left(\frac{\partial}{\partial z} \frac{1}{l}\right) d A
$$

The fields $B_{x}$ and $B_{y}$ are obtained similarly as follows:

$$
\begin{aligned}
& B_{X}=\frac{\mu_{0} I}{4 \pi} \int_{\dot{A}} \frac{\partial}{\partial z}\left(\frac{\partial}{\partial x} \frac{I}{l}\right) d A \\
& B_{Y}=\frac{\mu_{0} I}{4 \pi} \int_{A} \frac{\partial}{\partial z}\left(\frac{\partial}{\partial y} \frac{I}{l}\right) d A
\end{aligned}
$$

\section{APPLICATION TO MAGNET END}

A configuration of Fermilab-style magnet end is shown in Figure $2(a)$. The conductors are wound edgewise on to a mandrel. The field generated from the virtual coil penetrates the magnet winding. The radial field on a cylinder is taken through the winding and is integrated over the winding surface, as shown in Figure $2(b)$. The end winding geometry is taken into account for this integration. 
The field $B_{x}$ at $\left(X_{0}, Y_{0}, Z_{0}\right)$ is therefore written as

$$
\begin{aligned}
B_{x} & =\frac{\mu_{0} I}{4 \pi} \iiint \int \frac{\partial}{\partial r}\left(\frac{\partial}{\partial x} \frac{I}{l}\right) r d \theta d z \cdot N r d \phi d r \\
& =\frac{\mu_{0} I N}{4 \pi} \iiint \int r^{2} \frac{\partial}{\partial r}\left(\frac{\partial}{\partial x} \frac{I}{l}\right) d z d r d \theta d \phi
\end{aligned}
$$

where $N$ stands for winding density. The fields $B_{y}$ and $B_{z}$ are given similarly as

$$
\begin{aligned}
& B_{y}=\frac{\mu_{0} I N}{4 \pi} \iiint \int r^{2} \frac{\partial}{\partial r}\left(\frac{\partial}{\partial y} \frac{l}{l}\right) d z d r d \theta d \phi \\
& B_{z}=\frac{\mu_{0} I N}{4 \pi} \iiint \int r^{2} \frac{\partial}{\partial r}\left(\frac{\partial}{\partial z} \frac{I}{l}\right) d z d r d \theta d \phi
\end{aligned}
$$

The integration in Eq. (8), (9) and (10) should be done over all coils. in the magnet.

\section{CALCULATION EXAMPLE}

The field on the $\mathrm{Y}-\mathrm{Z}$ plane of the end winding is usually of our concern in Figure $2(a)$, and it is calculated in this example. One can see $B_{x}=0$ on the $\mathrm{Y}-\mathrm{Z}$ plane for the symetric reason. The field $\mathrm{B}_{\mathrm{Y}}$ on the $\mathrm{Y}-\mathrm{Z}$ plane is written as

$$
B_{\mathrm{Y}}=\frac{\mu_{0} I N}{4 \pi} \int_{\phi=\phi 1}^{\phi_{2}} \int_{\theta=\phi}^{\phi^{\prime}}\left[\mathrm{F}_{\mathrm{y}}\right]_{r, z} \mathrm{~d} \theta \mathrm{d} \phi
$$

where $\phi^{\prime}=\phi_{\text {sym }}+\left(\phi_{\text {sym }} \phi\right)$ and $\phi_{\text {sym }}$ is an angle of symmetry plane of the coil concerned. The function $\left[F_{Y}\right]_{r, z}$ is given as

$$
\left[F_{Y}\right]_{r, z}=\int_{r=r_{1}}^{r_{2}} \int_{z=-s}^{s} r^{2} \frac{\partial}{\partial r}\left(\frac{\partial}{\partial y} \frac{1}{l}\right) d z d r
$$

where $s=e+\sqrt{2} R(\sin \theta-\sin \phi)^{\frac{3}{2}} . \quad F_{y}$ can be expressed as follows: 


$$
\begin{aligned}
F_{y} & =\iint r^{2} \frac{\partial}{\partial r}\left(\frac{\partial}{\partial y} \frac{I}{\ell}\right) d z d r \\
& =\int r^{2} \frac{\partial}{\partial r}\left(\int \frac{\partial}{\partial y} \frac{1}{\ell} d z\right) d r \\
& =\frac{r^{2}}{\ell}-2 \int \frac{r}{\ell} d r \\
& =\left(z-z_{0}\right)\left\{\frac{l}{\ell} \frac{r^{2}}{\ell^{2}}\left(r \cdot \sin \theta-r_{0}\right)-2 \cdot \sin \theta \cdot \log \left|\ell+r-r_{0} \cdot \sin \theta\right|\right. \\
& \left.-\frac{r_{0} \cdot \cos 2 \theta}{1 z-z_{0} I} \log \left|\frac{\ell+1 z-z_{0} I}{\ell-1 z-z_{0} I}\right|+\frac{2 r_{0}}{I z-z_{0} I} \sin 2 \theta \cdot \tan ^{-1}\left(\frac{1 z-z_{0} I}{r_{0} \cos \theta} \frac{r-r_{0} \cdot \sin \theta}{\ell}\right)\right\}
\end{aligned}
$$

where $l_{r}=\left(r^{2}-2 r r_{0} \cdot \sin \theta+r_{0}{ }^{2}\right)^{\frac{1}{2}}$.

The $\mathrm{B}_{z}$ is written as follows:

$$
\begin{aligned}
& B_{z}=\frac{\mu_{0} I N}{4 \pi} \int_{\phi=\phi_{1}}^{\phi_{2}} \int_{\theta=\phi}^{\phi^{-}}\left[F_{z}\right]_{r, z} d \theta d \phi \\
& F_{z}=-\frac{r^{2}}{\ell}+2 \ell+2 r_{0} \sin \theta \cdot \log \left|\ell+r-r_{0} \sin \theta\right|
\end{aligned}
$$

A computational study was made on a three-shell quadrupole magnet $^{3}$ design with an operational field gradient of $50 \mathrm{kG} / \mathrm{i} \mathrm{n}$. Eq. (11) and (14) were applied to different types of the magnet ends, and the field on $\mathrm{Y}-\mathrm{Z}$ planes was computed. Figure 3 shows the configuration of the magnet end on the $Y-Z$ plane. The field on the innermost layer on each shell was calculated, and the locations are indicated by dots. The results obtained are shown in Figure 4. The peak field in each configuration is around $70 \mathrm{kG}$.

The configuration "a2" in Figure 3 was approximated by helix and arc Iines for input ${ }^{4}$ for GFUN. The field computed was found to be one percent lower than results given above. 
Taking into consideration the configuration for GFUN, input is slightly different from the original one; the disagreement is minimal. Since our purpose is to check the gross field features of the magnet ends, GFUN seems to be adequate.

\section{CONCLUSION}

1. Magnetic field calculation based on mutual inductance between magnet windings and virtual coil was presented, and it was shown how it is applied to a Fermilab-style magnet.

2. Although configuration of Fermilab-style magnet winding was approximated for GFUN input, GFUN gave a good agreement with the results obtained by the mutual inductance method.

\section{A.CKNOWLEDGEMENT}

The authors wish to acknowledge Drs. R.I.Turner and R.J.Lari of Argonne National Laboratory for their help to use GFUN.

\section{REFERENCES}

1. F.E.Mills and G.H.Morgan, "Particle Accelerators", 5, 227 (1973).

2. H.I.Rosten, Rutherford Laboratory Report RL-73-096, 1973.

3. K.Ishibashi and A.D.McInturff, "Stress Analysis of superconducting IOT Magnets for Synchrotron", presented at the 9 th International Cryogenic Engineering Conference, Kobe, Japan, May 1982.

4. A.G.Armstrong, C.J.Collie, N.J.Diserens, M.J.Newman, J.Simkin and C.W.Trowbridge, "GFUN3D Users Guide", Rutherford Laboratory Report RI-76-029/A, 1976. 


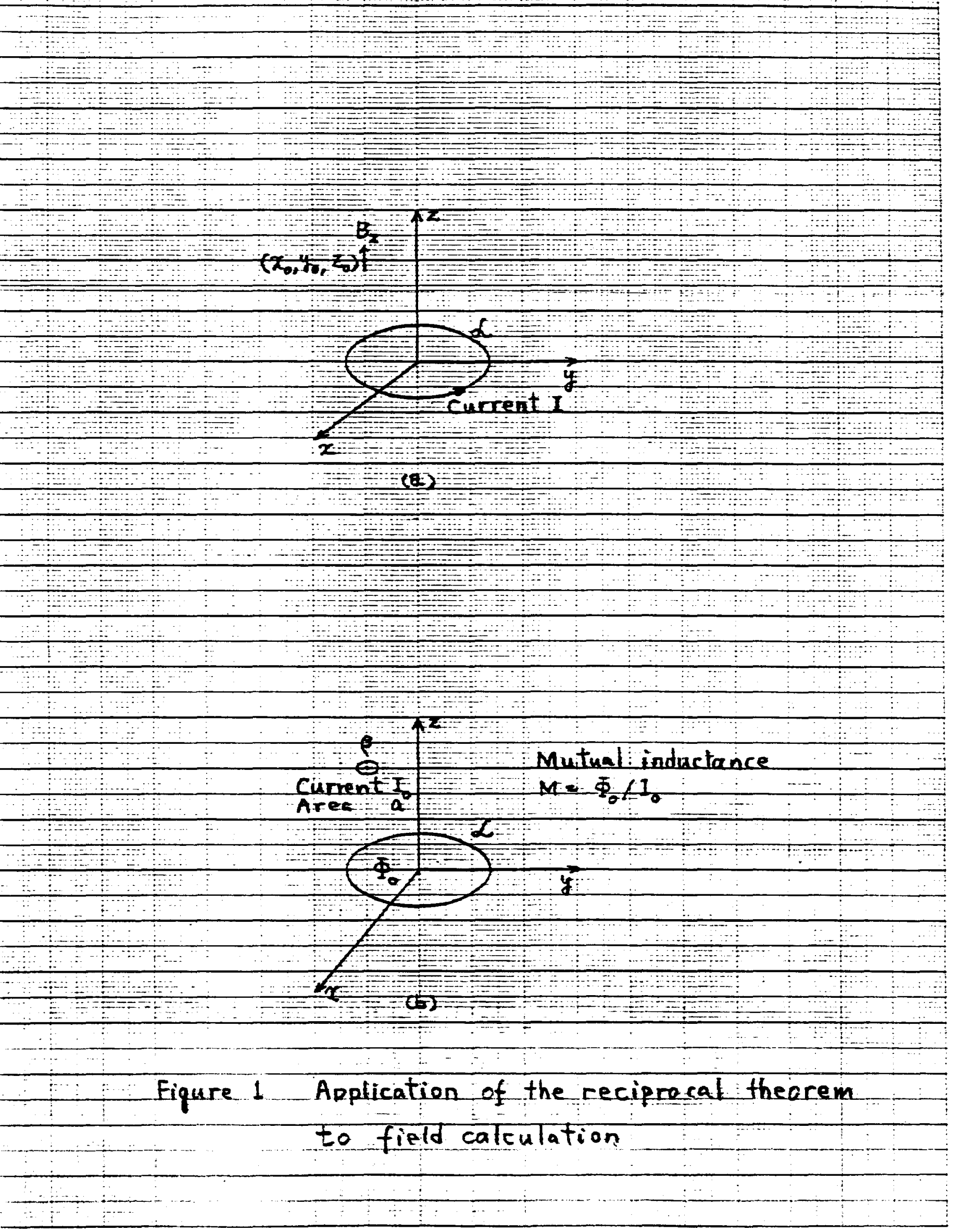




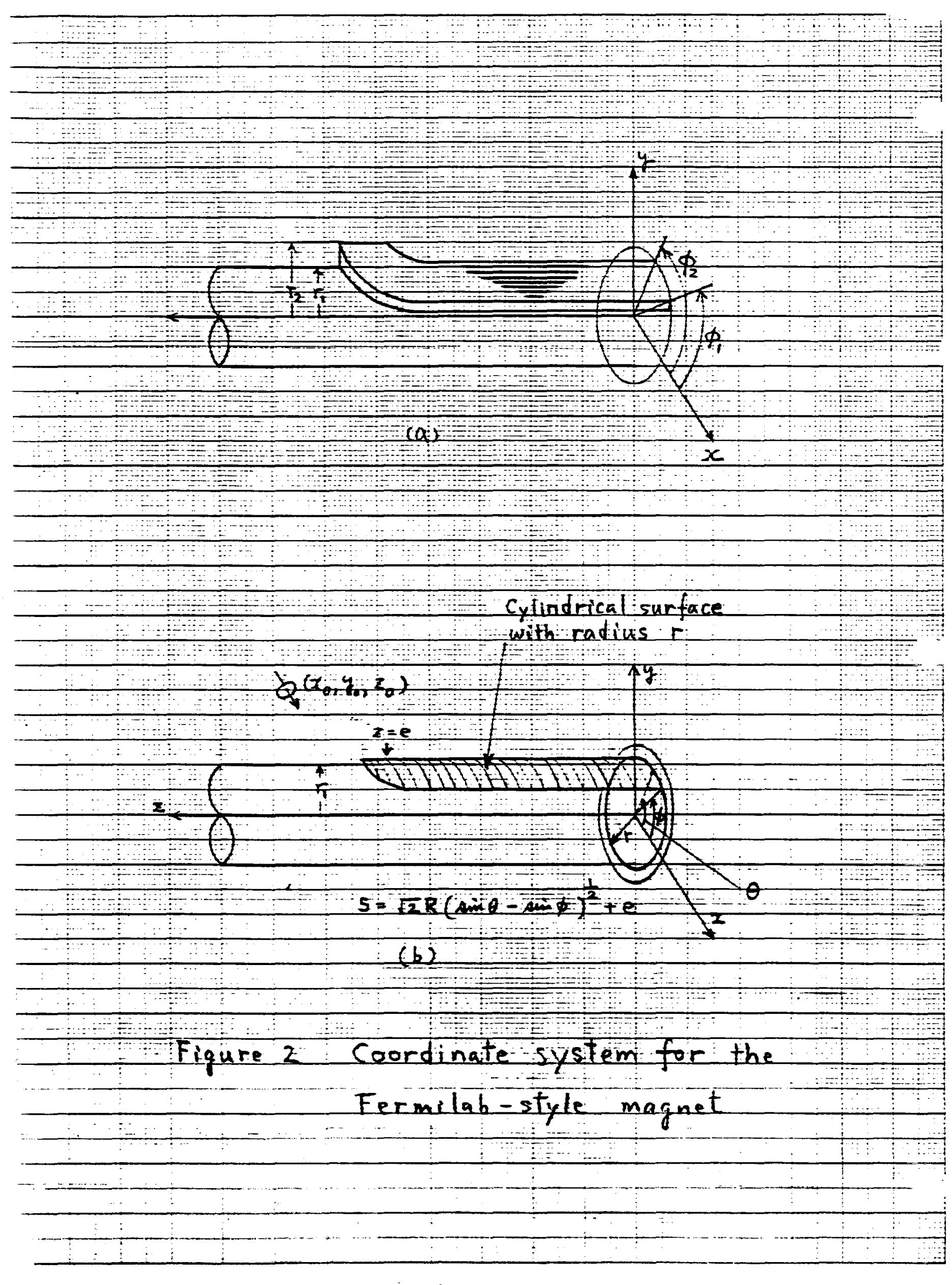




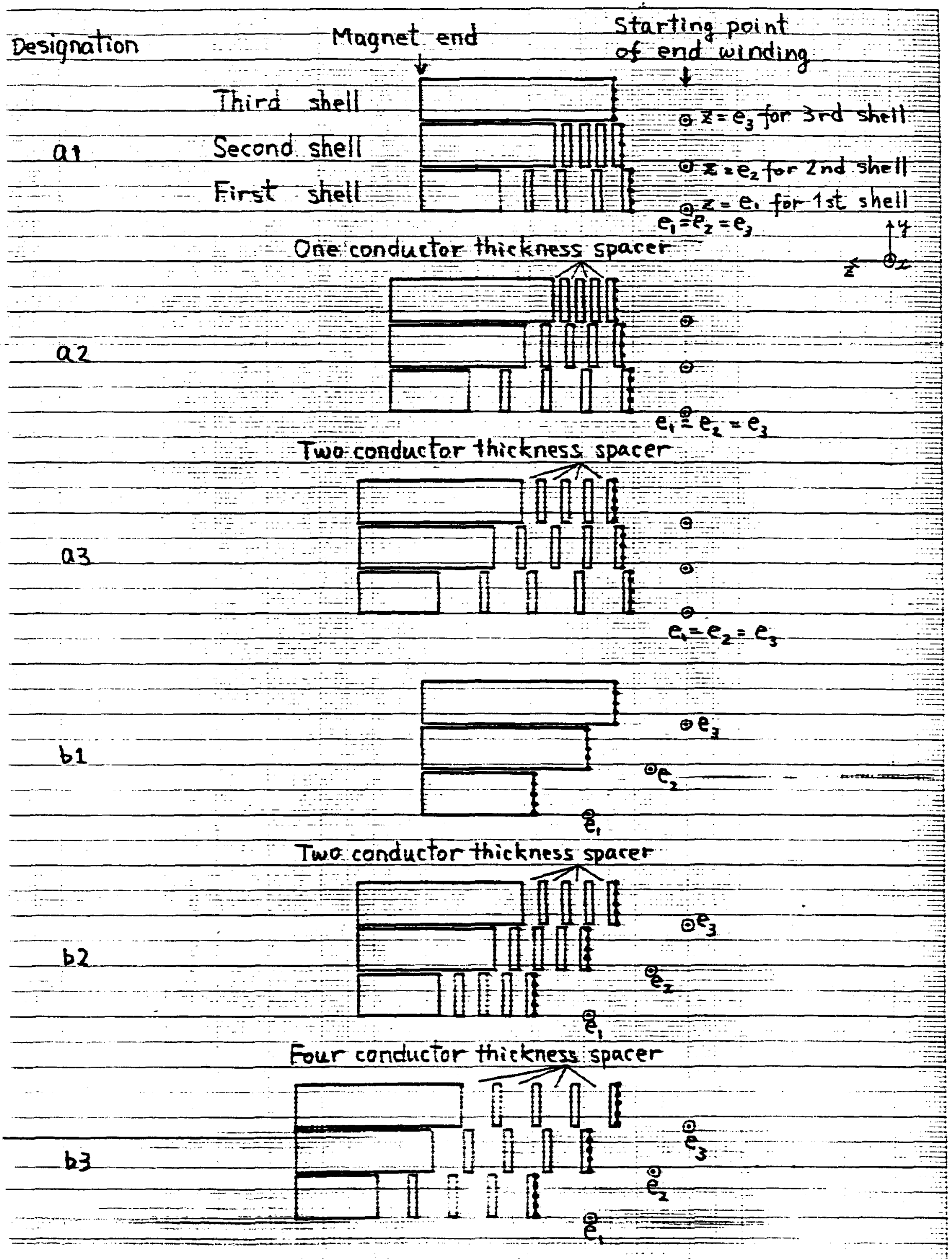

Figure 3 Crosssectional view of end winding on $Y=Z$ plane 


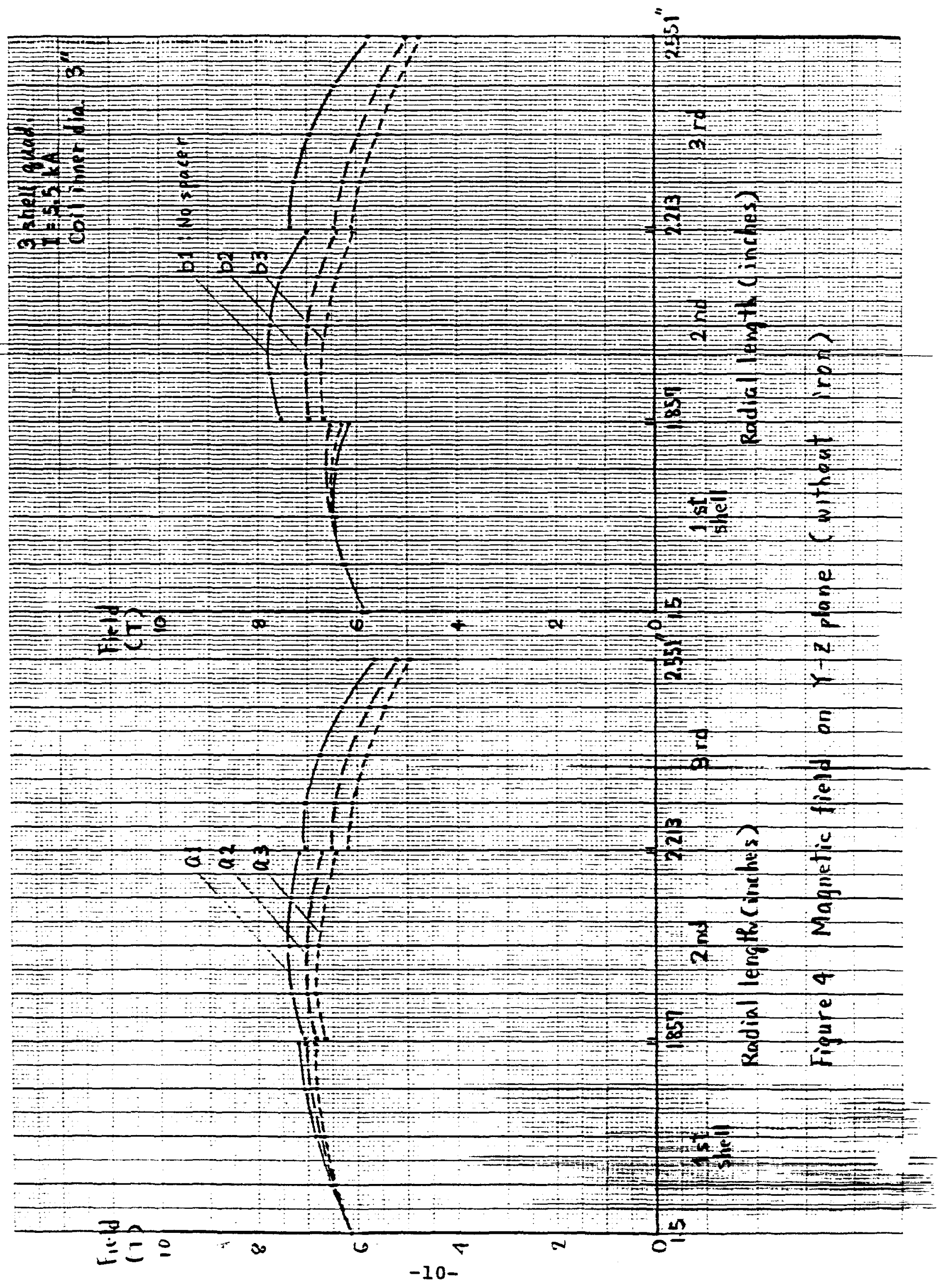

